\title{
Equilibrium and nonequilibrium solitons in a lossy split-step system with lumped amplification
}

\author{
Rodislav Driben and Boris A. Malomed \\ Department of Interdisciplinary Studies, \\ Faculty of Engineering, Tel Aviv University, Tel Aviv 69978, \\ Israel
}

November 3, 2018

\begin{abstract}
We propose a more realistic version of the recently introduced split-step model (SSM), which consists of periodically alternating dispersive and nonlinear segments, by adding uniformly distributed loss and lumped gain to it. In the case when the loss is exactly balanced by gain, a family of stable equilibrium solitons (ESs) is found. Unless the system's period $L$ is very small, saturation is observed in the dependence of the amplitude of the established ES vs. that of the initial pulse. Stable nonequilibrium solitons (NESs) are found in the case when the gain slightly exceeds (by up to $\simeq 3 \%$ ) the value necessary to balance the loss. The existence of NESs is possible as the excessive energy pump is offset by permanent radiation losses, which is confirmed by computation of the corresponding Poynting vector. Unlike ESs that form a continuous family of solutions, NES is an isolated solution, which disappears in the limit $L \rightarrow 0$, i.e., it cannot be found in the overpumped nonlinear Schrödinger equation. Interactions between ESs turn out to be essentially the same as in SSM without loss and gain, while interactions between NESs are different: two NESs perturb each other by the radiation jets emanating from them, even if they are separated by a large distance. Moving NESs survive collisions, changing their velocities.
\end{abstract}




\section{Introduction}

It is commonly known that the observation and use of solitons in various systems, such as fiber-optic telecommunication links [1], makes it necessary to periodically compensate losses. If the system is long, which is the case for telecommunication links, or if it has the shape of a closed loop which is composed of different elements (examples are fiber lasers generating the so-called stretched pulses [2], and a ferromagnetic film combined with an amplifier, which is able to support periodic trains of propagating magnetic solitons [3]), the loss-compensating gain is usually provided for by short (effectively, point-like) amplifying elements which are periodically placed (lumped) in the case of the long link.

If the system itself, except for the lumped amplifiers, is uniform, the discreteness of the gain is not an important factor: in that case, it is enough to demand that the gain averaged along the link is in balance with the uniformly distributed loss. In particular, if the undamped uniform nonlinear system supports solitons, it is easy to demonstrate that the balance condition provides for the stable existence of solitons in the presence of loss and amplification 画. On the other hand, a number of strongly heterogeneous systems, based on periodic alternation of very different elements, have been recently introduced, chiefly in the context of nonlinear optics. These include the well-known dispersion management (i.e., alternation of fiber segments with opposite signs of the group-velocity dispersion [5]), one- or multidimensional tandem systems concatenating linear and quadratically nonlinear waveguide sections [6], media composed of alternating layers with different values of the Kerr coefficient [7], alternate waveguides, in which waveguiding and anti-waveguiding nonlinear segments are juxtaposed [8], dynamical lattices subject to diffraction management [9], and some others.

A new system belonging to this class was recently proposed in the form of a split-step model (SSM) [10]. In SSM, pieces of a dispersive optical fiber with negligible nonlinearity (in fact, these are not necessarily fibers; they may instead be short elements with a very strong group-velocity dispersion, such as a fiber Bragg grating [11] or a photonic crystal [12]) periodically alternate with nonlinear fiber segments operating close to the zero-dispersion point, in which the dispersion is negligible (these may be replaced by short elements in which strong effective Kerr nonlinearity is created by means of the cascading mechanism in quadratically nonlinear optical crystals [13).

In the limit when lengths of both dispersive and nonlinear segments are much smaller than the soliton's 
dispersion length, the SSM model is tantamount to the commonly known split-step algorithm simulating the nonlinear Schrödinger (NLS) equation [14]. However, on the contrary to the split-step algorithm, in SSM the separation between dispersive and nonlinear segments is a physical feature, rather than a numerical trick, so that lengths of the segments are not small, being comparable to or larger than the dispersion length of a pulse (soliton) that may propagate in the system.

In the above-mentioned works, it has been demonstrated, by means of direct numerical simulations, that, in the absence of loss, all those strongly inhomogeneous periodic systems support very robust quasisoliton pulses, although it may be quite difficult to prove their existence and stability in a rigorous form. The introduction of losses and compensating gain poses a new problem for solitons in these systems, the most fundamental issue being whether the straightforward lumped amplification, with the average gain compensating the loss, may support stable soliton-like pulses.

The objective of the present work is to look for stable solitons in what seems to be the simplest and, possibly, most fundamental model of the type described above: SSM with uniform losses and periodically placed point-like amplifiers. Very recently, it has been demonstrated that, in a more general case, when the average gain considerably exceeds the loss rate (by several per cent), SSM solitons cannot be stable [15]. It was found that, nevertheless, efficient stabilization of solitons can be achieved by means of synchronous intensity modulators (these time-domain control elements are used in fiber-optic systems [16]).

In the present work, we focus on a more special case, that, by itself, may be less relevant to direct applications to telecommunications, but appears to have a fundamental interest for the understanding of pulse dynamics. It is a case when the gain exactly compensates the loss in SSM, or the relative excess gain

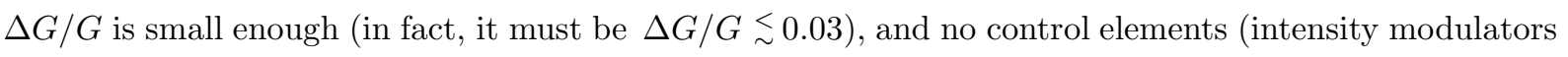
or frequency-domain filters) are added. We find stable solitons of two different types. First, in the case when the average gain exactly compensates the loss $(\Delta G=0)$, we find stable equilibrium solitons (ESs). Usually, stationary solitary pulses in dissipative systems, supported by the balance between loss and gain, are isolated solutions, typical examples being an exact unstable solitary-pulse solution to the cubic complex Ginzburg-Landau (GL) equation [17 and its stable counterpart (also an exact solution) in a system of linearly coupled cubic and linear GL equations [18]. A difference of ESs found in the present model is that they form a continuous family, which may be parameterized by their amplitude. 
More accurately, the amplitude $\eta_{\text {fin }}$ of the soliton in the established state is nearly equal to the initial amplitude $\eta_{\text {in }}$, provided that $\eta_{\text {in }}$ is not too large. For larger values of $\eta_{\text {in }}$, saturation in the dependence $\eta_{\text {fin }}\left(\eta_{\text {in }}\right)$ is observed.

If small excess gain is present, being limited to the above-mentioned interval $\Delta G / G \lesssim 0.03$, stable nonequilibrium solitons (NESs) are found. Unlike the equilibrium ones, they are indeed isolated solutions with a uniquely defined value of the amplitude. Their existence in the presence of the excess gain is explained by the fact that they permanently emit small-amplitude radiation waves. We check that the size of the corresponding Poynting vector (energy flux) exactly matches the rate of the energy pump into the soliton.

In this paper, we also demonstrate the existence of stable moving ESs and NESs in the same model, and consider collisions between them, which turn out to be inelastic in the latter case, although NESs survive collisions. Interactions between immobile solitons are also considered, with a conclusion that NESs strongly perturb each other, via the radiation jets that they continuously emit.

In fact, ESs are quite simple pulses, which smoothly go over into the usual nonlinear Schrödinger (NLS) solitons as the system's period is vanishing. On the other hand, NESs appear to be novel solitary pulses which are supported by the stable balance between gain, dissipative loss, and the emission of radiation. NESs have no counterparts in the usual uniform NLS equation: we demonstrate that they disappear if the SSM period vanishes (they disappear too if the period becomes too large). They also disappear if the loss parameter is very large, and they only exist within the above-mentioned narrow margin of values of the excess gain.

\section{The model}

The linear lossy dispersive segment of the system is described by the damped linear Schrödinger equation,

$$
i u_{z}+\frac{1}{2} u_{\tau \tau}=-\alpha_{D} u
$$

In an optical fiber, $u, z$ and $\tau$ are, respectively, the local amplitude of the electromagnetic waves, propagation distance, and local time $(\tau \equiv t-z / V$, where $t$ and $V$ are the time proper and mean group velocity of the wave packet), the dispersion coefficient is normalized to be 1 , and $\alpha_{D}>0$ is a loss constant. 
Obviously, Eq. (1) can be solved by means of the Fourier transform in $\tau$ (which is used as a part of the standard split-step numerical algorithm [14]).

The nonlinear segment of the system is governed by the dispersionless NLS equation,

$$
i u_{z}+|u|^{2} u=-i \alpha_{N} u
$$

where the nonlinearity coefficient is normalized to be 1 , and $\alpha_{N}>0$ is the loss constant in the nonlinear segment (we assume that the third-order dispersion may be neglected). A solution to Eq. (2) is obvious,

$$
u(z, \tau)=u(0, \tau) \exp \left(-\alpha_{D} z\right) \exp \left[i \frac{|u(0, \tau)|^{2}}{2 \alpha_{N}}\left[1-\exp \left(-2 \alpha_{N} z\right)\right]\right.
$$

We define the system's elementary cell as an interval between midpoints of two neighboring nonlinear segments. Point-like amplifiers are set at junctions between cells. They acts on the wave field so that

$$
u(\tau) \mapsto u(\tau) \cdot e^{G},
$$

where $G$ is the gain (the gain defined in terms of the signal's power and measured in $\mathrm{dB}$ is $8.69 G$ ). Thus, the full transformation (map) for the pulse passing a cell can be represented as a superposition of two nonlinear phase transformations (3) corresponding to the nonlinear half-segments at the cell's edges, and the Fourier transform corresponding to the dispersive segment in the middle of the cell, which are followed by the multiplication as per Eq. (雨).

In order to prevent radiation from re-entering the integration domain, the linear equation (11) was solved directly by means of numerical methods in a broad integration interval $\Delta T$, placing absorbers at its edges. In fact, the absorbers emulate a real physical effect, namely, the fact that the energy emitted with radiation is lost.

If the lengths of the dispersive and nonlinear segments are $L_{D}$ and $L_{N}$, the cell size is $L=L_{D}+L_{N}$. Note that the left-hand sides of Eqs. (11) and (2) are separately invariant with respect to transformations

$$
\tau \rightarrow \tau / \Lambda_{D}, z \rightarrow z / \Lambda_{D}^{2} \text {, and } u \rightarrow \Lambda_{N} u, z \rightarrow z / \Lambda_{N}^{2}
$$

The rescalings (5) may be used (with $\Lambda_{D}^{2} / \Lambda_{N}^{2}=L_{D} / L_{N}$ ) to make $L_{D}=L_{N} \equiv L / 2$, which we assume below to hold.

With regard to the normalizations adopted, an average (distributed) version of the model amounts to 
a perturbed NLS equation,

$$
i u_{z}+\frac{1}{4} u_{\tau \tau}+\frac{1}{2}|u|^{2} u=-i\left[\frac{1}{2}\left(\alpha_{N}+\alpha_{D}\right)-\frac{G}{L}\right] .
$$

The balance between the loss and gain is expected in the case when the right-hand side of Eq. (6) vanishes, i.e., when the gain takes the equilibrium value

$$
G_{\mathrm{eq}}=\frac{L}{2}\left(\alpha_{N}+\alpha_{D}\right)
$$

\section{Equilibrium solitons}

As it was demonstrated in Ref. [10], SSM without loss and gain gives rise to a family of stable solitary pulses. The first objective of the present work is to generate similar pulses in the model introduced in the previous section, in the case when the loss and gain are balanced as per Eq. (7). To this end, we simulated the evolution of initial pulses that were taken as fundamental solitons of the unperturbed NLS equation (6),

$$
u(z=0, \tau)=\eta \operatorname{sech}(\eta \tau)
$$

with an arbitrary amplitude $\eta$.

In a vast parametric range, the initial pulse (8) readily generates a solitary wave, which remains stable as long as simulations could be run, see a typical example in Fig. 1. As is seen in the figure, the pulse changes its shape against the initial configuration (8). Detailed analysis shows that the established pulse very well fits to the expression (8), but with a different (smaller) value of the amplitude. As well as the fundamental NLS soliton, the established pulse has no chirp (i.e., no intrinsic phase structure [1]).

The family of equilibrium solitons, alias ESs [the ones subject to the equilibrium condition 母)] found this way is characterized by a dependence of the final ("output") value $\eta_{\text {out }}$ of the amplitude on the initial ("input") amplitude $\eta_{\text {in }}$, which is presented, for different fixed values of the period $L$, and for different values of the loss constant, in Figs. 2(a) and 2(b). As is seen, for small values of $\eta_{\text {in }}$ the amplitude $\eta_{\text {out }}$ is virtually identical to it. However, with the increase of $\eta_{\text {in }}$ the growth of $\eta_{\text {out }}$ slows down, and, eventually, $\eta_{\text {out }}$ saturates at a constant value $\eta_{\text {sat }}$, that strongly depends on $L$, but very weakly on $\alpha$, see Fig. 2(b). An example of the formation of a stable ES with the amplitude $\eta_{\text {out }}$ which is much smaller than $\eta_{\text {in }}$ (in the case of strong saturation) is displayed in Fig. 3. 
Figure 2(a) shows that, in the limit of very small $L$, the saturation does not take place, and in this case we simply have $\eta_{\text {out }}=\eta_{\text {in }}$, which has a simple meaning: for small $L$, the averaging applies, transforming the SSM with the balanced loss and gain into the unperturbed NLS equation [see Eq. (6)]. In the latter equation, the initial pulse (8) generates a fundamental soliton with the amplitude $\eta$.

We stress that different branches of the dependence shown in Fig. 2(a) were not aborted arbitrarily: except for the one corresponding to $L=0.1$, they all terminate at points corresponding to a maximum value of $\eta_{\text {in }}$ for which the initial pulse (8) can generate a soliton. If $\eta_{\text {in }}$ exceeds this maximum value, the initial pulse blows up, generating spatio-temporal "turbulence". We did not study the latter regime in detail, focusing on the regular behavior.

\section{Nonequilibrium solitons}

Numerical simulations of the model demonstrate that, if the gain slightly exceeds the equilibrium value (7), stable solitons are still generated in a narrow interval of values of the excess gain: $\left(G-G_{\text {eq }}\right) / G_{\text {eq }} \lesssim 0.03$. A typical example of the formation of such a nonequilibrium soliton (NES) is shown in Fig. 4, which corresponds to $\left(G-G_{\mathrm{eq}}\right) / G_{\mathrm{eq}}=0.024$. If the relative excess gain exceeds the maximum value $\simeq 0.03$, the system blows up.

The apparent existence of the stable pulse shown in Fig. 4 suggests a natural question: how is the energy balance provided for in this case, if the gain exceeds the loss? An answer is that the energy pump into NES by the excess gain is compensated by extra loss in the form of small-amplitude radiation waves emitted by the pulse. Indeed, the background on top of which the NES showed in Fig. 4 is found is not just $u \equiv 0$, but a small-amplitude wave field.

To analyze this issue, we define the norm of the solution (it is what is usually called energy in optics), $E=\int_{-\infty}^{+\infty}|u(z, \tau)|^{2} d \tau$. The energy is an obvious dynamical invariant in the case of the unperturbed NLS equation, as well as in the SSM without loss and gain. In the present model, the evolution of the energy of a localized pulse is governed by a balance equation. After straightforward calculations which use integration by parts, the balance equation can be cast in the form

$$
\frac{d E_{\mathrm{sol}}}{d z}=2\left[G \sum_{n} \delta(z-n L)-\alpha(z)\right] E_{\mathrm{sol}}-\left.D(z) \cdot \operatorname{Im}\left(u^{*} u_{\tau}\right)\right|_{\tau=-T} ^{\tau=+T} .
$$

Here, the soliton's energy is defined as the integral over some finite interval, $-T<\tau<+T$, which 
is essentially larger than a proper size of the soliton, but smaller than the total size of the domain between the above-mentioned edge absorbers, $E_{\mathrm{sol}} \equiv \int_{-T}^{+T}|u(z, \tau)|^{2} d \tau$. The last term in Eq. (9), which is produced by the integration by parts, represents the Poynting vector (energy flux) of the field $u$ at the points $\tau= \pm T$. Lastly, Eq. (9) assumes that the amplifiers are placed at the points $z=L n$, the coefficient $\alpha(z)$ takes values $\alpha_{N}$ and $\alpha_{D}$ in the nonlinear and dispersive segments, respectively, while $D(z) \equiv 1$ inside the dispersive segments, and $\equiv 0$ inside the nonlinear ones.

In the case when the coefficients $\alpha$ and $G$ are small, Eq. (9) can be averaged over a large number of cells, reducing to

$$
\frac{d E_{\mathrm{sol}}}{d z}=\left[2 L G-\left(\alpha_{N}+\alpha_{D}\right)\right] E_{\mathrm{sol}}-\frac{1}{2}\left(J_{+}+J_{-}\right)
$$

where $J_{ \pm}$are $z$-averaged values of $\operatorname{Im}\left(u^{*} u_{\tau}\right)$ at the points $\tau= \pm T$. Taking, for instance, the NES displayed in Fig. 4, and assuming that its shape is exactly described by Eq. (8), we find $E_{\text {sol }}=3.844$, hence the first term on the right-hand side of Eq. (10), i.e., the net rate of the energy pump into the NES, is 0.0165 . On the other hand, numerical data yield the value $\left(J_{+}+J_{-}\right) / 2 \approx 0.0155$ of the second term (the net energy flux across the points $\tau= \pm T$ ). The comparison shows reasonable agreement between the energy pump and emission rates; the remaining difference may be explained by a deviation of the exact shape of the field from the expression (8).

A drastic difference of NES from ES is that NES is an isolated solution, which is found with a single value of its amplitude, while ESs form a continuous family. In other words, NES is a unique attractor in the system.

It is instructive to summarize data showing the NES amplitude as a function of the period $L$ and dissipative constant $\alpha$, see Fig. 5. An important inference, suggested by Fig. 5(a) and confirmed by many more simulations, is that the amplitude diverges (i.e., the NES blows up) as $L \rightarrow 0$. This result means that NES cannot exist in the uniform NLS equation with unbalanced gain, which is quite obvious by itself. Thus, we conclude that the existence of NES is a distinctive feature of SSM. Note that NES does not exist (actually, it blows up) past the point where the curve terminates in Fig. 5(b). 


\section{Interactions and collisions between solitons}

Interactions between solitons is the next natural issue to consider. To this end, we note that, although Eq. (2) and, hence, SSM as a whole, are not Galilean invariant, moving pulses can be readily generated from the zero-velocity ones considered above, "pushing" them by means of a multiplier exp $(-i \chi \tau)$ with an arbitrary real constant $\chi$, added at the point $z=0$, which gives rise a soliton's velocity proportional to $\chi[10]$. The result is formation of stable moving solitons, both ESs and NESs, see an example of moving NESs below in Fig. 7. Apparently, the amplitude of a moving NES is somewhat smaller than that of its quiescent counterpart, but this issue was not studied in detail.

Simulations demonstrate that both the interaction between quiescent ESs, as well as collision between moving ones, are almost identical to those which were studied in detail in SSM without loss and gain in Ref. 10]. Namely, collisions are quasi-elastic, and two zero-velocity ESs do not interact, provided that the separation between them exceeds a certain minimum value.

The situation is different in the case of NESs. First, even if the separation between two zero-velocity NESs is large, they strongly perturb each other - not via direct interaction, but rather by the radiation "jets" emitted by each soliton, see a typical example in Fig. 6. Note that each soliton has a smaller amplitude than in isolation. Second, moving NESs survive collisions, but, as it is seen in the example displayed in Fig. 7, the pulses reappear after the collision with different values of the velocity [in fact, the slope of their trajectories in the $(\tau, z)$ plane increases], and with a smaller amplitude. However, the amplitude gradually relaxes to the unique value selected by NES moving at a given velocity.

\section{Conclusion}

We have added loss and gain to a model of a long periodic system consisting of separated dispersive and nonlinear segments. It was shown that, if the loss is exactly balanced by gain, a family of stable equilib-

rium solitons exists. Unless the system's period is very small, saturation is observed in the dependence of the amplitude of the established soliton vs. that of the initial pulse. Stable nonequilibrium solitons were found in the case when the gain slightly exceeds (by up to $\simeq 3 \%$ ) the value necessary to balance the loss. The existence of NESs is possible as the excessive energy pump is offset by permanent radiation losses, which is confirmed by computation of the corresponding Poynting vector. Unlike ESs that exist 
as a continuous family of solutions, NES is an isolated one, and it disappears when the system's period vanishes. Interactions between ESs were found to be essentially the same as in the model without loss and gain, while interactions between NESs are different: two NESs perturb each other by their radiation jets, even if they are separated by a large distance. Moving NESs survive collisions, with a change of the velocity.

\section{Acknowledgements}

We appreciate useful discussions with M.J. Ablowitz and P.L. Chu. 


\section{References}

[1] A. Hasegawa and Y. Kodama. Solitons in optical communications (Oxford University Press: Oxford, UK, 1995).

[2] H.A. Haus, K. Tamura, L.E. Nelson, and E.P. Ippen, IEEE J. Quant. Electr. 31 (1995) 591.

[3] G.A. Melkov, A.A. Serga, V.S. Tiberkevich, Y.V. Kobljanskij, and A.N. Slavin, Phys. Rev. E 63 (2001) 066607; G.A. Melkov, Y.V. Kobljanskyj, A.A. Serga AA, V.S. Tiberkevich, and A.N. Slavin, Phys. Stat. Sol. A 189 (2002) 1007.

[4] Yu.S. Kivshar and B.A. Malomed, Rev. Mod. Phys. 61 (1989) 763.

[5] J.H.B. Nijhof, N.J. Doran, W. Forysiak and F.M. Knox, Electron. Lett. 33 (1997) 1726.

[6] L. Torner, IEEE Photon. Techn. Lett. 11 (1999) 1268; L. Torner, S. Carrasco, J.P. Torres, L.-C. Crasovan, and D. Mihalache, Opt. Commun. 199 (2001) 217.

[7] L. Bergé, V.K. Mezentsev, J. Juul Rasmussen, P.L. Christiansen, and Yu.B. Gaididei, Opt. Lett. 25 (2000) 1037; I. Towers and B.A. Malomed, J. Opt. Soc. Am. B 19 (2002) 537.

[8] A. Kaplan, B.V. Gisin, and B.A. Malomed, J. Opt. Soc. Am. B 19 (2002) 522.

[9] M.J. Ablowitz and Z.H. Musslimani, Phys. Rev. Lett. 87 (2001) 254102.

[10] R. Driben and B.A. Malomed, Opt. Commun. 185 (2000) 439 (2000); ibid. 197 (2001) 481.

[11] G. Lenz, B.J. Eggleton, C.K. Madsen, and R.E. Slusher, IEEE J. Quant. Electr. QE37 (2001) 525.

[12] M. Notomi, K. Yamada, A. Shinya, J. Takahashi, C. Takahashi, and I. Yokohama, Phys. Rev. Lett. 87 (2001) 253902.

[13] L.J. Qian, X. Liu, and F.W. Wise, Opt. Lett. 24 (1999) 166; X. Liu, L. Qian, and F. Wise, Opt. Lett. 24 (1999) 1777.

[14] A.C. Newell and J.V. Moloney. Nonlinear Optics (Addison-Wesley|: 1992).

[15] R. Driben, A. Orenstein, and B.A. Malomed, Opt. Commun. 205 (2002) 139. 
[16] K. Suzuki and M. Nakazawa, Opt. Fiber Techn. 1 (1995) 289 (1995); T.Yamamoto and M. Nakazawa, Opt. Lett. 26 (2001) 647 (2001); E. Pincemin, O. Audouin, B. Dany, and S. Wabnitz, IEEE J. Light. Techn. 19 (2001) 624.

[17] N.R. Pereira and L. Stenflo, Phys. Fluids 20 (1977) 1733.

[18] J. Atai and B.A. Malomed, Phys. Lett. A 246 (1998) 412. 


\section{$7 \quad$ Figure captions}

Fig. 1. An example of the establishment of a stable pulse of the equilibrium type in SSM with the balanced loss and gain $\left[\alpha_{N}=\alpha_{D}=0.035, L=1\right.$, and, in accord with Eq. (7), $\left.G=0.035\right]$. The initial pulse is taken as the NLS soliton (8) with $\eta=1$. In this and subsequent figures, $|u|^{2}$ ("power") is shown vs. $\tau$ at the end of each cell.

Fig. 2. The established ("output") amplitude of the equilibrium soliton vs. the initial ("input") amplitude. In (a), $\alpha_{N}=\alpha_{D}$ is fixed to be 0.035 ; in (b), $L$ is fixed to be 1 , and $\alpha_{N}=\alpha_{D} \equiv \alpha$.

Fig. 3. Formation of a stable equilibrium soliton in the case when Fig. 2 shows strong saturation. The initial amplitude in Eq. (8) is $\eta=4, L=1, \alpha_{N}=\alpha_{D}=0.035$.

Fig. 4. An example of the formation of a nonequilibrium soliton. The amplitude of the input pulse and the SSM's parameters are the same as in the case shown in Fig. 1, except for $G=0.03585$, which corresponds to the relative excess gain $\left(G-G_{\text {eq }}\right) / G_{\text {eq }} \approx 0.024$.

Fig. 5. The amplitude of the stable nonequilibrium soliton vs. the system's period ("stepsize") $L$ (a) and the loss parameter $\alpha_{N}=\alpha_{D} \equiv \alpha$ (b). In both panels, the relative excess gain is fixed to be 0.024 ; in (a), $\alpha=0.035$, and in (b), $L=1$.

Fig. 6. Interaction of two nonequilibrium solitons (the same ones as in Fig. 4). The initial separation between them is $\Delta \tau=18$, the full-width-at-half-maximum [1] of each one being initially $T_{\mathrm{FWHM}}=2$. (a) The distribution of $|u(z, \tau)|^{2}$; (b) the same shown by means of level contours.

Fig. 7. An example of the collision between two nonequilibrium solitons. Each moving soliton is generated from the quiescent one shown in Fig. 4, multiplying it by $\exp ( \pm i \tau / 2)$. The side view of the

distribution of $|u(z, \tau)|^{2}$ is displayed [in this case, a full three-dimensional picture like those in Figs. 1, 3, 4, and 6(a) would seem messy], the inset showing trajectories of centers of the two solitons on the plane $(\tau, z)$. 
This figure "Fig1.jpg" is available in "jpg" format from: http://arxiv.org/ps/nlin/0207026v1 
This figure "Fig2.jpg" is available in "jpg" format from: http://arxiv.org/ps/nlin/0207026v1 
This figure "Fig3.jpg" is available in "jpg" format from: http://arxiv.org/ps/nlin/0207026v1 
This figure "Fig4.jpg" is available in "jpg" format from: http://arxiv.org/ps/nlin/0207026v1 
This figure "Fig5.jpg" is available in "jpg" format from: http://arxiv.org/ps/nlin/0207026v1 
This figure "Fig6.jpg" is available in "jpg" format from: http://arxiv.org/ps/nlin/0207026v1 
This figure "Fig7.jpg" is available in "jpg" format from: http://arxiv.org/ps/nlin/0207026v1 